\title{
New in the marketplace
}

The following table contains a summary of product launches and approvals identified in international markets in recent weeks.

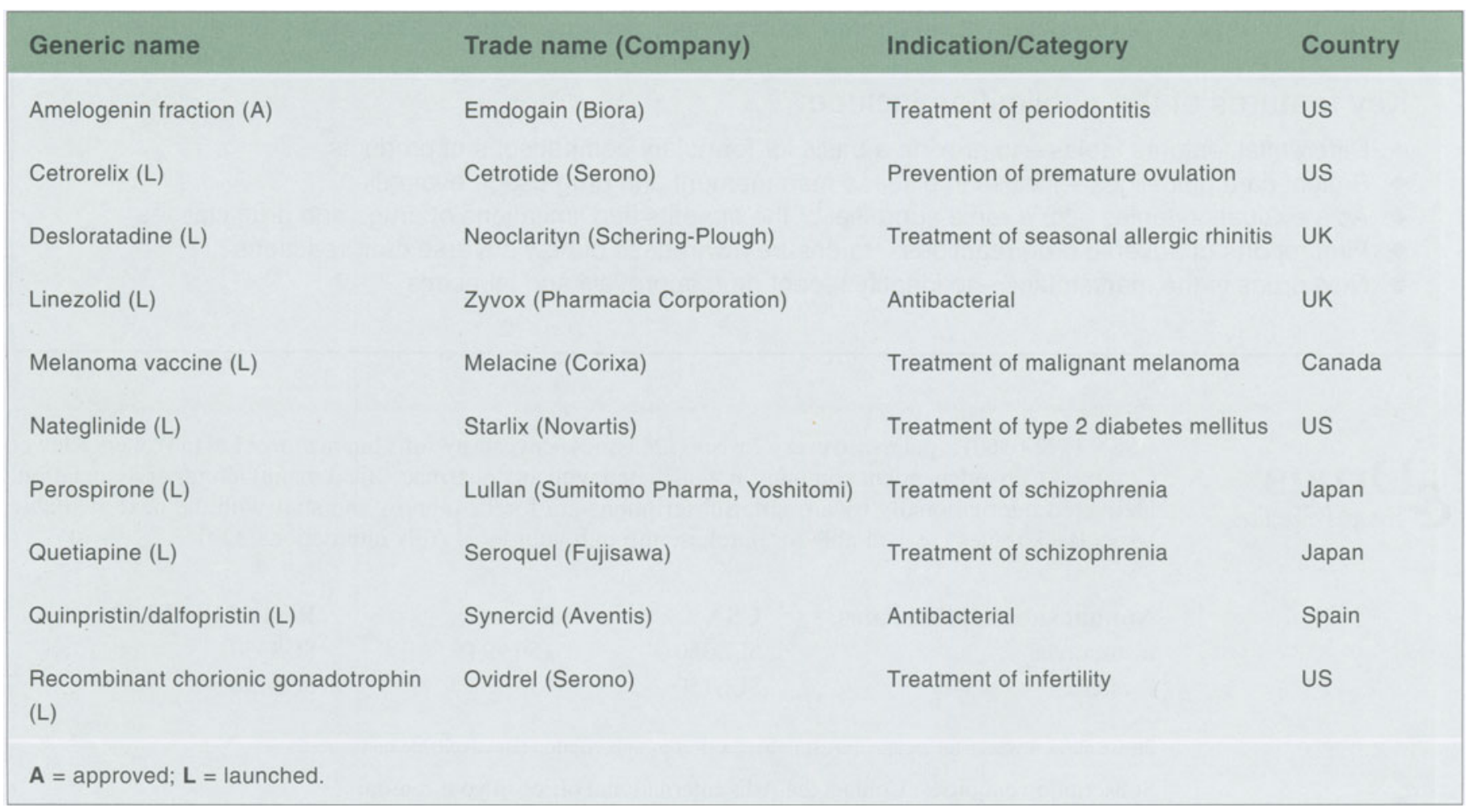

\section{Addendum}

In the article 'Which is the best aromatase inhibitor for postmenopausal breast cancer?' [Drug Ther Perspect 2000 Oct 11; 16 (8): 5-7], exemestane is referred to as an androgenic aromatase inhibitor. Exemestane is actually an aromatase inactivator. Anti-aromatase agents fall into 2 classes that act by different mechanisms, the irreversible steroidal inactivators (formestane and exemestane), and the reversible nonsteroidal inhibitors (aminoglutethimide, anastrazole, letrozole and fadrozole). The interaction between nonsteroidal aromatase inhibitors and aromatase is reversible and inhibitors may be competitively displaced from the binding site, allowing the aromatase enzyme to resume conversion of androgens to estrogens. On the other hand, the steroidal inactivators compete with androstenedione at the active site of the enzyme, and are then transformed to an intermediate that binds irreversibly to aromatase, causing it to be inactivated permanently. ${ }^{[1]}$

The article states that exemestane may be associated with androgenic effects. Androgenic events have been reported in some patients receiving once daily exemestane $200 \mathrm{mg}$, but these were rarely reported at the recommended daily dosage $(25 \mathrm{mg})$. Exemestane is the only steroidal inactivator with a once-daily oral dosage regimen that irreversibly inhibits conversion of androgens to estrogens. ${ }^{[1]}$

\section{Reference}

1. Clemmett D, Lamb HM. Exemestane: a review of its use in postmenopausal women with advanced breast cancer.

Drugs 2000 Jun; 59 (6): 1279-96 